\title{
Sentido de identidad comunitaria, empoderamiento y participación política en estudiantes de la UNMSM
}

\section{Sense of community identity and political participation in UNMSM students}

\author{
John Omar Espinoza Quiroz ${ }^{1}$ \\ Universidad Científica del Sur \\ María de las Mercedes Raguz Zavala ${ }^{2}$ \\ Pontificia Universidad Católica del Perú
}

\section{Resumen}

El presente estudio tuvo como propósito profundizar en la experiencia subjetiva de un grupo de integrantes de los Centros de Estudiantes de la UNMSM, con relación a su participación política en el semestre académico 2019-II, durante el conflicto social producto de la construcción de un bypass en la avenida Venezuela, en el Cercado de Lima, ciudad de Lima, en octubre de 2019. Las entrevistas individuales fueron transcritas y analizadas con el programa Atlas.ti. Desde un enfoque cualitativo y un diseño fenomenológico, los testimonios derivaron en cinco categorías y las representativas fueron contrastadas con el sentido de identidad comunitaria, empoderamiento y participación política. Se encontró que los estudiantes consideran relevante contar con apoyo personal y sentir un contento personal, por los aprendizajes obtenidos en convivencia con su comunidad universitaria, los que expresan desde su participación en ámbitos académicos, políticos y de relaciones interpersonales. Esta práctica se vincula con un empoderamiento individual y organizacional que dialoga con una participación política institucionalizada. Por último, se concluye que la participación política es transversal a su vida universitaria y, en este contexto, toda participación estudiantil en la universidad se considera como política.

Palabras claves: Sentido de identidad comunitaria; empoderamiento; participación política estudiantil; análisis fenomenológico.

\footnotetext{
1 Docente en la Universidad Científica del Sur.

Autor para correspondencia: jespinozaqu@ucientifica.edu.pe ORCID: https://orcid.org/0000-0001-6641-0000

2 Docente y Decana de la Facultad de Psicología en la Pontificia Universidad Católica del Perú.

E-mail: mraguz@pucp.pe ORCID: https://orcid.org/0000-0001-9539-7220

(C) Los autores. Este artículo es publicado por la Revista de Investigación en Psicología de la Facultad de Psicología, Universidad Nacional Mayor de San Marcos. Este es un artículo de acceso abierto, distribuido bajo los términos de la licencia Creative Commons Atribucion - No Comercia_Compartir Igual 4.0 Internacional. (http://creativecommons.org/licenses/by-nc-sa/4.0/) que permite el uso no comercial, distribución y reproducción en cualquier medio, siempre que la obra original sea debidamente citada.
} 


\begin{abstract}
The purpose of this study was to deepen the subjective experience of a group of members of some of the UNMSM student centers, regarding their political participation in the academic term 2019-II, during the social conflict caused by the construction of a bypass on Venezuela avenue, in the center of Lima city, in October 2019. The interviews were transcribed and analyzed using the Atlas.ti computer program. From a qualitative approach and a phenomenological design, the testimonies derived in five categories and the representative ones were contrasted with the sense of community identity, empowerment, and political participation. It was found that the students consider it relevant to have personal support and feel personal contentment for the learning acquired in coexistence with their university community, which they express during their participation in academic, political and interpersonal relations fields. This practice is associated to individual and organizational empowerment, which links to institutionalized political participation. It is concluded that political participation is transversal to university life and, in this context, student participation in the university is always considered as political.
\end{abstract}

Keywords: Sense of community identity; empowerment; student political participation; phenomenological analysis.

En la actualidad, las referencias de estudios orientados a la comprensión del fenómeno de la participación política en estas comunidades estudiantiles se dividen en investigaciones cuantitativas que buscaron conocer características individuales de los dirigentes políticos en relación a la personalidad, el liderazgo, los rasgos de autoritarismo y la participación convencional y no convencional (Córdova, 2013; Jorge, 2016), y, por otro lado, investigaciones cualitativas que se orientaron a conocer historias de vida de dirigentes estudiantiles, registro documental de la historia del movimiento estudiantil y percepciones hacia la política y las instituciones (Cano, Quiroz y Nájar, 2017; Minedu, 2014; Yarasca, 2019).

Según lo revisado en Brussino, Rabbia y Sorribas (2008) y Sandoval, Hatibovic y Bobowik (2018), existen diferentes perspectivas del estudio de la participación política. Tenemos la perspectiva de la legalidad, entendida como acción política convencional. Es decir, la participación política que respeta el orden de las instituciones y las normas que subyacen a la estructura de poder tradicional, donde participar está permitido bajo ciertos requisitos. La acción política no convencional, por otro lado, sería aquella que interrumpe el orden, que, mayormente, va en sentido contrario al statu quo de la participación bajo el régimen y el modelo electoral, que valora diferentes manifestaciones individuales y/o colectivas para ejercer ciudadanía. Esta propuesta dicotómica es propia de la psicología política, la antropología social y la sociología política, las mismas que han aportado de forma notable a la comprensión de la participación política juvenil (Botero y Torres, 2008).

Hatibovic, Sandoval y Cárdenas (2012) señalan que esta tipología de la participación se puede entender en dimensiones: participación política y ciudadanía; 
participación política como la acción de un voluntariado, y participación política y movilización social. La primera se refiere a la indagación de la participación en un ámbito de ejercicio ciudadano y deber cívico. La segunda es una expresión voluntaria de la participación y, en ese sentido, una oportunidad de desarrollo del estudiante autónomo e íntegro. La última dimensión destaca las vivencias, las historias de vida, la diversidad en las expresiones de participación en una subjetividad social.

Por otro lado, es conocido que la participación en sí misma es un acto de ejercicio del poder (Ferullo, 2006). En ese sentido, la participación ejercida por los individuos en un grupo se orienta hacia el empoderamiento. De acuerdo con Sánchez-Vidal (2007), el empoderamiento significa la tenencia del poder progresivo, determinado por el control de diferentes recursos a diferentes niveles de la persona en interacción con su entorno. También se debe resaltar el aporte de la teoría multinivel de Zimmerman (Fernández, Morales y Molero, 2012; Silva y Martínez, 2004), que específica que el empoderamiento opera en tres niveles: individual, organizacional y comunitario, que le otorga el rasgo de proceso dinámico.

Según Puddifoot (2003), el sentido de identidad comunitaria es la orientación psicológica del individuo a la comunidad y se puede dividir en aspectos personales y aspectos compartidos. Los aspectos personales son los relacionados con el sentido de apoyo personal (SMS), sentido de contento personal (SPC), sentido de inclusión personal activa (SPI); mientras que los aspectos compartidos se orientan al sentido activo de compromiso personal (PCE), sentido de vecindad (SV) y estabilidad percibida (EP).

Cabe precisar que la caracterización de cada dimensión fue validada por Puddifoot (2003), de la Universidad de Luton, Inglaterra, con la intención de extender la explicación del modelo de sentido de comunidad de McMillan y Chavis, de la década de 1980, que solo consistía en cuatro dimensiones: membrecía, satisfacción de necesidades, capacidad de influencia y conexión emocional (Puddifoot, 2003).

En relación con nuestro marco epistemológico fenomenológico, en psicología se han realizado investigaciones para explorar y comprender las dimensiones manifiestas de la experiencia subjetiva en el individuo, relacionados con sus interacciones sociales (Lozano, 2008; Tarazona, 2004). La experiencia subjetiva es la dimensión que agrupa un sistema de ideas, actitudes, sentimientos, percepciones y expectativas de un individuo sobre una vivencia particular (Kaliksztein, 2001). Asimismo, "es una trama de relaciones intersubjetivas, construido y reconstruido histórica y socialmente, que articula una diversidad de dimensiones" (Grimberg, 2001, p.45).

\section{CONTEXTO}

El objetivo inicial de esta investigación fue el describir la experiencia subjetiva sobre la participación política de un grupo de estudiantes de la Universidad 
Nacional Mayor de San Marcos (UNMSM), en el contexto de las diferentes incidencias que conllevaron el proceso de reforma universitaria actual, iniciada en 2014, con la publicación de la nueva Ley Universitaria (Ley n. ${ }^{\circ} 32220$ ) y la creación de la Sunedu (Superintendencia Nacional de Educación Superior Universitaria), en 2015, que es la institución del Estado peruano que supervisa el cumplimiento de las condiciones básicas de calidad educativa superior en las universidades públicas y privadas del país.

Es de particular relevancia destacar el contexto del recojo de datos de la presente investigación, que cobra un sesgo particular al coincidir, incidentalmente, con una situación de movilización de la participación estudiantil sanmarquina, al activarse un conflicto que tenía once años en letargo. El conflicto del bypass de la avenida Venezuela, según las crónicas e imágenes difundidas por los medios de comunicación local, se inició en 2008, como una propuesta de la administración municipal de Lima Metropolitana, en ese entonces liderada por el alcalde Luis Castañeda Lossio que, en diálogo con las autoridades de la UNMSM, buscaba mejorar el tránsito en ese sector de la capital y brindar seguridad a los estudiantes que utilizaban estas vías para acceder al campus de la ciudad universitaria. A raíz de expresiones de disconformidad de un sector de estudiantes de la comunidad sanmarquina, la obra quedaría paralizada por más de una década.

En setiembre de 2019, en vista de que la nueva autoridad municipal de Lima deseaba retomar las gestiones para concluir la obra pendiente, los conflictos volvieron a manifestarse con diferentes actores, por parte de las autoridades de la universidad y sus estudiantes. Es, en este contexto no previsto, que se realiza ta recolección de información. Al revivirse el conflicto e involucrarse la representación estudiantil de diferentes maneras, los datos recogidos presentan este importante y valioso sesgo, ya que activan la participación de manera significativa, lo que facilita una riqueza de experiencias vivas a compartir.

\section{MÉTODO}

\section{Tipo y diseño de investigación}

La investigación es de tipo cualitativa, pues se orienta a la comprensión de los hechos desde la perspectiva de sus participantes (Martínez, 2004). Asimismo, cuenta con un diseño de estudio fenomenológico basado en el Interpretative Phenomenological Analisys (Larkin y Thompson, 2012; Willig, 2013).

\section{Participantes}

Los participantes del estudio fueron representantes estudiantiles de dos facultades de la UNMSM en Lima. Existen diferentes estudios y publicaciones sobre esta comunidad, en contextos político-sociales diversos, interesados en profundizar sobre las características de sus protagonistas (Jave, Cépeda y Uchuypoma, 2015; Minedu, 
2014; Montoya, 2016; Nureña, 2016, 2017; Yarasca, 2019), los que relevan que la UNMSM se ha caracterizado por albergar una comunidad estudiantil que, además de estar formada por profesionales, ha tenido y tiene participación política en el escenario local y nacional en diferentes momentos de la historia democrática del Perú.

Se seleccionaron dos facultades: Ciencias Sociales y Letras y Ciencias Humanas, porque en nuestra revisión de literatura, los estudiantes de estas carreras cuentan con conocimientos y prácticas vinculadas con espacios de decisión política. De forma adicional, en la experiencia personal como estudiante sanmarquino, he sido testigo de las principales expresiones de acción política no convencional originadas en la ciudad universitaria y, específicamente, en estas facultades situadas alrededor de la entrada principal de la universidad.

En cuanto a estos, se buscaron estudiantes voluntarios que pudieran brindar experiencias autorreportadas en participación política en, como mínimo, el último año calendario. La participación se basó en la accesibilidad (Penalva, Alaminos, Francés y Santacreu, 2015) y buscó una heterogeneidad que brindara la mayor riqueza de información posible, según Patton (2014). Se entrevistaron cuatro hombres y cinco mujeres entre 19 y 32 años, matriculados en el semestre 2019-II, y que tenían un cargo de representación en la gestión del Centro de Estudiantes de sus respectivas escuelas profesionales, en su facultad.

Todos los participantes fueron debidamente informados del propósito del estudio y se contó con su consentimiento, incluyendo la aceptación para la validación de las transcripciones y para la publicación de sus testimonios. Además, se cuidó la confidencialidad con el uso de dos códigos.

\section{Instrumento}

Se eligió la entrevista en profundidad, recomendada para el uso de investigaciones cualitativas (Izcara y Andrade, 2003; Hernández-Sampieri y Mendoza, 2018; Katayama, 2014). Se diseñó una guía de entrevista en profundidad, validada por jueces expertos y aprobada, así como el protocolo de investigación, también aprobada el Comité de Ética de la Investigación de la Pontificia Universidad Católica del Perú (PUCP).

\section{Recojo y análisis de información}

De acuerdo con Izcara y Andrade (2003), se llevó a cabo una recolección de información de ensayo con jóvenes representantes estudiantiles de otra institución educativa superior, para el ajuste temático y de preguntas de la guía principal de recolección de datos.

El recojo de testimonios definitivo se realizó durante el semestre 2019-II. Las sesiones de entrevista se coordinaron por medio telefónico y de manera presencial, 
a fin de contactar directamente con los representantes de los Centros de Estudiantes y presentar el estudio, de acuerdo con lo que dictan los procedimientos éticos y los criterios de calidad para estudios cualitativos de credibilidad-autenticidad propuestos en (Noreña, Alcaraz-Moreno, Rojas y Rebolledo-Malpica, 2012).

Se realizaron 18 sesiones de entrevistas, del 26 de agosto al 15 de octubre de 2019, y el 7 y 8 de enero de 2020, coincidiendo, como se señaló antes, con el conflicto del bypass. Cada entrevista individual con los participantes de la UNMSM requirió dos sesiones de 30 a 40 minutos de duración. Todas fueron registradas en audio, debidamente transcritas en formato Word, y compartidas de forma virtual con los participantes para su validación y autenticación. Se diseñó un etiquetado de transcripciones para facilitar el análisis de datos.

El procesamiento se llevó a cabo mediante técnicas de análisis temático y de contenido, con el programa Atlas ti versión 8.0 (Penalva et al., 2015), procedimiento centrado en la comprensión de las estructuras que son base del discurso de los participantes. Se entiende la identificación de estas estructuras como el resultado de una reflexión y discusión de diferentes miradas respecto a un mismo fenómeno (Braun y Clarke, 2006; Martínez, 2004; y Vasilachis de Gialdino, 2014). Los pasos utilizados se pueden resumir en categorización de la información (codificación y preanálisis); diseño de uno o más mapas conceptuales que integran categorías, y construcción de la interpretación, que consiste en establecer relaciones entre categorías que se fundamentan en hipótesis de trabajo emergentes que se contrastan con el marco teórico referencial y las reflexiones individuales.

\section{RESULTADOS}

\section{Categorización de la información}

El resultado de la categorización significó elegir 86 citas de las entrevistas seleccionadas, mediante los procedimientos de codificación abierta y codificación por lista disponibles en Atlas ti (Penalva et al., 2015).

Un primer producto de este proceso de exploración fueron las experiencias vividas por cada uno de los participantes. Esta se logró al identificar, en sus testimonios, hechos relacionados con los conceptos participación, participación estudiantil y participación política. Los mismos nos permitieron obtener un total de 86 citas entre las nueve entrevistas analizadas.

Después de ingresar las categorías preestablecidas: sentido de identidad comunitaria (conceptos explicados en la introducción) y las categorías emergentes: expresiones de la participación política y vivencia personal en la participación política, puede apreciarse la frecuencia con la que fueron citados estos temas en el autorreporte de los participantes en la tabla 1. 
Es importante precisar que, si bien la cantidad absoluta de citas es 86 , muchas de ellas fueron codificadas con más de una categoría en diferentes ocasiones, ya que el testimonio de los participantes contiene diferentes temas a la vez (análisis temático). En ese sentido, la columna de frecuencia de citas (enraizamiento) expresa otra sumatoria: 152 .

Tabla 1

Frecuencia de citas de categorías

\begin{tabular}{lc}
\hline Nombre de categoría & Enraizamiento \\
\hline Expresiones de la participación política (PP) & 50 \\
Actitud hacia la política (AP) & 31 \\
Sentido de identidad comunitaria (SIC) & 18 \\
Motivaciones para la participación política & 15 \\
Vivencia personal en participación & 13 \\
Expresiones de participación estudiantil & 10 \\
Opinión pública/estigma social & 8 \\
Estudios previos/familia de origen & 7 \\
Total & 152 \\
\hline
\end{tabular}

\section{Mapas conceptuales y construcción de la interpretación}

Se diseñó un mapa conceptual en el que se utilizó la herramienta Administrador de Redes del programa. En este se incluyeron todas las categorías disponibles (categorías preestablecidas y emergentes) para realizar la triangulación de testimonios con la interpretación de su contenido en la misma interfaz que el programa facilita. De forma adicional, se diseñó un mapa conceptual sin la asistencia del programa, donde se resaltaron los conceptos de nuestro marco teórico referencial, los mismos que se conectaron con sus propias dimensiones al utilizar como argumento el testimonio de los participantes.

Para la presente publicación, se ha tomado en cuenta el análisis de los testimonios vinculados con las categorías: expresiones de la participación política (categoría emergente), sentido de identidad comunitaria (categoría preestablecida), vivencia personal en participación (categoría emergente), expresiones de participación estudiantil (categoría emergente) y opinión pública/estigma social (categoría emergente), las mismas que se agruparon en el análisis cualitativo de los resultados en tres supracategorías: sentido de identidad comunitaria, empoderamiento y participación política. Para ello, recurriremos a la selección de citas representativas, tomando en cuenta que las supracategorías mencionadas, de acuerdo con el marco teórico referencial, coexisten en el ámbito de reflexión teórico-práctico de la psicología comunitaria (Ferullo, 2006; Montero, 2011), enfoque que subyace a la propuesta de este estudio. 
En la figura 1 se muestra nuestra hipótesis de trabajo central para el análisis cualitativo de las categorías y supracategorías, de acuerdo con los testimonios recogidos:

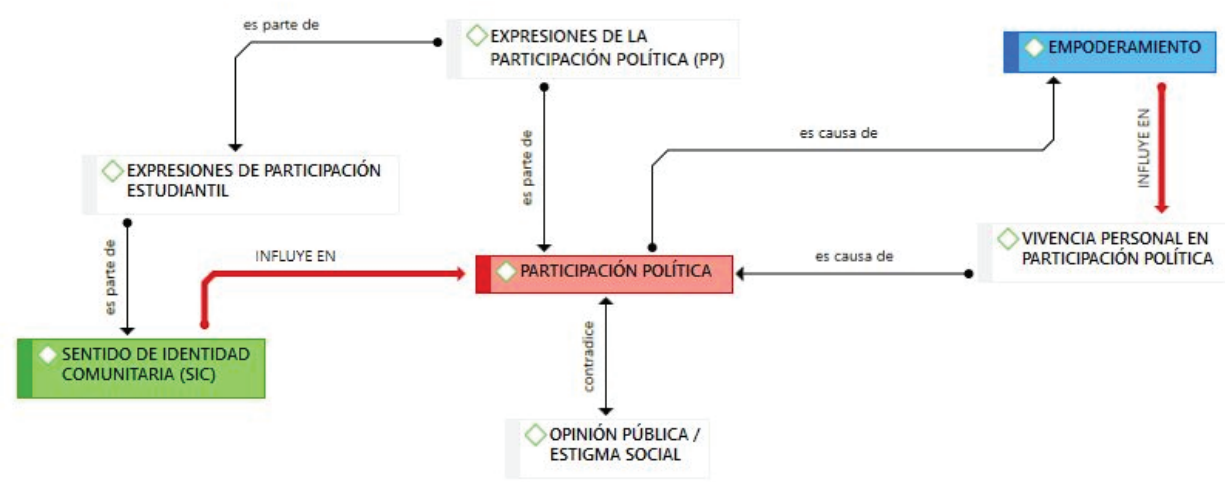

Figura 1. Mapa conceptual: relación y categorías y supracategorías.

Elaboración propia utilizando interfaz de Atlas.ti.

\section{Sentido de identidad comunitaria}

En esta sección encontraremos los testimonios asociados con acciones y verbalizaciones que involucran un vínculo afectivo, y orientadas a un grupo de estudiantes pares en la universidad. Estos se pueden representar como compañeros de estudios de la misma escuela académica profesional, facultad o universidad.

He sido indiferente a la escuela, a mi CEAR, y considero que, por esa parte, pude haber hecho más cosas. Pude haber participado más, apoyando más y no lo hice conscientemente. (...) Más que todo, trato de que este ciclo ya de pensar en mi escuela, en mi carrera más que todo. (E1, sesión de entrevista, 26 de agosto de 2019)

En esta primera cita se evidencia, por un lado, el reconocimiento de un sentimiento de lejanía personal o desinterés por parte de su grupo, como es la escuela académica profesional. También se observa el anhelo de haber participado de manera más activa en las necesidades compartidas con esta. De acuerdo a lo mencionado por Puddifoot (2003), decimos que este estudiante describe la importancia que tiene para su comunidad, de la que se siente parte y comparte un sentido de inclusión personal activa, pero que a su vez cuenta con la necesidad pendiente de demostrarlo.

Podría decir que yo era una de las chicas que menos participaba, menos, menos participaba en mi base. Por ejemplo, en el Centro de Estudiantes no me involucraba. En mi base asumí comité económico en primer ciclo, pero de ahí me alejé. (E8, sesión de entrevista, 15 de octubre de 2019) 
De lo vertido en este testimonio, notamos que la estudiante valora su participación compartida con los compañeros de promoción como de forma limitada, que esta se expresó solo al inicio de su carrera y se orientó al apoyo de acciones específicas. Asimismo, expresa un distanciamiento después de estas primeras colaboraciones. Según Puddifoot (2003), se considera que su participación habría iniciado con un sentido de apoyo personal, porque sentía que colaboraba con su grupo. Con esto podemos reflexionar que en el presente su experiencia de participación política, siendo más activa, como representante estudiantil, inició limitada, pero tuvo un impulso en el transcurso de su vida universitaria y que valoraría desde un sentido activo de compromiso personal:

Yo era una persona muy tímida, tenía miedos acá, entonces, participar, sí, poco a poco me ayudó bastante a conocer gente, a tener bastantes compañeros, a entender el compañerismo, (...), por ejemplo, "préstame la parrilla, esa parrilla. No es mía, es de unos compañeros y no me cobran nada". Entonces, ese vínculo no lo conocía, es algo muy bonito que agradezco bastante. (E8, sesión de entrevista, 15 de octubre de 2019)

En el mismo sentido de valorar lo positivo que ha sido su experiencia de participación política, en relación con su sentimiento de pertenencia con la comunidad universitaria, tenemos la siguiente cita:

Yo valoro que esto me ha hecho madurar, (...) y mejorar personalmente. No me di cuenta que tenía dentro estas habilidades hasta que ya pude, este, digamos, representar, y eso también me ha dado una responsabilidad, este, mayor, porque siento mucha presión, pero a la vez me gusta, me gusta sentir la presión. Me di cuenta de que, no, que, prefiero pensar colectivamente que individualmente. (E3, sesión de entrevista, 16 de setiembre de 2019)

En esta cita se observa un sentido de contento personal por las experiencias vividas y aprendizajes obtenidos. Estos son valorados como positivos, a su vez, son invertidos en el presente mediante una actitud de responsabilidad frente al cargo que ocupa y lo que esto representa. Este aspecto personal es importante para complementar la mirada del sentido de identidad comunitaria con los aspectos compartidos, muy a pesar de que estos no se manifiesten o sean percibidos como ausentes.

No hay Centro Federado hasta el momento (...) Es lo mismo que le está pasando a la Escuela de Sociología y a otras escuelas de Ciencias Sociales: no hay Centros Federados porque no (han) habido las elecciones todavía (...) No, tienen otras fechas (...), desde el año pasado (...) no, no habido transitorio. Bueno, la transitoria, en este caso, es cada Centro de Estudiantes (...), así como las juntas transitorias del Centro de Estudiantes lo asumen los delegados de cada base, por así decirlo. (E5, sesión de entrevista, 27 de agosto de 2019) 


\section{Empoderamiento}

Como hemos visto con anterioridad, este proceso psicosocial de aprendizaje se considera en sí un ejercicio de poder, el mismo que puede ser analizado desde diferentes niveles, como los que propone la teoría multinivel de Zimmerman (Fernández et al., 2012; Silva y Martínez, 2004). A continuación, presentamos los testimonios de participación política más resaltantes.

Tenemos la participación política que se orienta al desarrollo personal de los estudiantes (nivel individual de empoderamiento) y que adquiere un valor para el individuo, el hecho de su propia práctica. Es así que en este testimonio se evidencia cómo su experiencia de participación se fue nutriendo de diferentes aspectos:

Fue una participación pasiva, más de solo ir a actividades. No me involucré directamente en política. Me pusieron nervioso porque me permitió explorar, más o menos, como era todo aquí. Primero, conocí asambleas, pero no participaba. (...) De a pocos me empecé a meter un poco más, fueron como más exploratorios, como más, también eran cuestiones más teóricas, más así, no tanto de la práctica. (E4, sesión de entrevista, 27 de agosto de 2019)

Según Zimmerman (como se citó en Fernández et al., 2012; Silva y Martínez, 2004), en el nivel organizacional de empoderamiento, las personas se encuentran con personas que orientan su participación política a generar cambios en su entorno estructural inmediato. De la experiencia recogida, tenemos la referencia a la acción de "cogobierno" en la toma de decisiones del ámbito académico en las facultades como la acción que más acerca al estudiante al sentimiento de fortalecimiento de la representación estudiantil en su propia facultad:

La Junta Directiva tiene que estar con el Comité de Gestión, (...), sí o sí, alumno del tercio superior, y no habían muchos que querían, porque no quieren arriesgar su puntaje académico, supongo (...), es que así está en nuestro estatuto. Entonces (...), ellos son los que trabajan con la escuela, a nivel ya de escuela (...), si, si, o sea, si no pasa por el comité de gestión, no puede ser validado. Es como la representación estudiantil a un nivel un poco más. (E3, sesión de entrevista, 16 de setiembre de 2019)

Para el nivel de empoderamiento comunitario, encontramos los testimonios que reflejan la necesidad de orientar la participación política general por parte de la comunidad universitaria, para el logro del fortalecimiento del movimiento estudiantil sanmarquino. El primer paso, para ello, sería poner en práctica estrategias para motivar la participación estudiantil en sus diferentes expresiones (académica, artística, deportiva, cultural, otros), ya que en el proceso de ejercer su participación desarrollarán y/o fortalecerán capacidades como colectivo, como el compromiso por las necesidades "en común" (Montero, 2011). Las estrategias 
iniciales para promover esta participación se encuentran en el ámbito académico y se trata de la promoción de foros académicos, seminarios, congresos nacionales e internacionales, pasantías, exposiciones, talleres, cursos cortos, entre otros.

Es desarrollar diferentes actividades y, sobre todo, charlas, conferencias, conversatorios referentes a la participación, la necesidad de la participación estudiantil dentro de la escuela y de la facultad, para que esto no quede en un trabajo aislado. El compromiso que tenemos es ese: realizar diferentes actividades para poder generar el interés de participación estudiantil en el desarrollo político de la universidad misma y, así, poder darle la posta a una junta electa. (E5, sesión de entrevista, 27 de agosto de 2019)

De acuerdo a la teoría multinivel del empowerment, si analizamos este en relación con las expresiones de participación política, podemos decir que la segunda se orienta a los diferentes niveles o dimensiones de la primera, para promover, en un nivel individual, principalmente, el desarrollo personal del estudiante. En el nivel organizacional se orienta a la búsqueda de consolidar la representación estudiantil en el cogobierno de sus facultades. En el nivel comunitario se espera el fortalecimiento de toda la comunidad universitaria sanmarquina.

\section{Participación política}

En la categoría central de análisis de este estudio, iniciaremos esta exploración de vivencias, considerando citas que hacen explícita su participación política en la universidad.

En este caso, yo no me involucro mucho en política porque tengo familia que está involucrada en política. Entonces, en lo personal, yo tengo firmado un compromiso notarial, el cual no me permite involucrarme demasiado en política, tiene ciertas cláusulas (...) Eso es porque mis familiares anteriores han sido bien revoltosos aquí en San Marcos (...) No, mis padres no, pero sí mi prima es sanmarquina. (E2, sesión de entrevista, 11 de setiembre de 2019)

Cuando analizamos la participación política, también nos corresponde analizar su ausencia, y por qué puede ser también limitada. Estas dos opciones que, en consecuencia, indican que la participación política en la universidad tiene un modo particular de influir en el comportamiento de toda la comunidad universitaria. En el caso de la cita anterior, se evidencia que la estudiante cuenta con una serie de restricciones que limitan su participación en la universidad, en su facultad y ante sus compañeros de la comunidad universitaria. Si bien esta restricción puede venir de un plano privado, como es de las decisiones de su propia familia que tiene influencias sobre otras esferas de su actuar en la universidad, hay un análisis propio de los estudiantes que se suma a este caso, y que explica el porqué de las dificultades para promover una participación política sostenible en la universidad. 
Yo creo que sí, incluso, me pasaba mucho cuando recién ingresaba. En el primer año, justamente, ese momento la mayoría viene con todos sus prejuicios del colegio, de la casa y, si pasaba cuando hablábamos, por ejemplo, del Centro Federado o de la federación, cómo era, como que si van a hacer protestas, no, de seguro deben ser rojos, esas cosas, o mucho miedo cuando hacen movilización de trabajadores. (E4, sesión de entrevista, 27 de agosto de 2019)

Se advierten fenómenos psicosociales presentes, como la estigmatización de que las acciones de participación política se derivan en alternativas de participación no convencional o en oposición a la norma. Esto ha generado, en el imaginario social, la percepción de que los jóvenes universitarios que participan en política no tienen ningún beneficio. En la experiencia de los estudiantes entrevistados, ellos describen con detalle que el pensamiento que tiene la sociedad sobre ellos los representa con características vinculadas a la transgresión del orden cotidiano.

Las primeras experiencias, donde los estudiantes asumen un rol más activo de participación política en la universidad, se enmarcan en lo normativo y convencional, lo aceptado por las reglas internas de la misma como institución educativa.

Me proponen hacerlo, no tenía mucha experiencia, como ya tenía un poco el bicho, (...), ya había visto desde afuera esto, decidí aceptar y ahí es donde empiezo a participar de verdad, a partir del Comité Electoral yo y tres compañeros de mi base más. (E4, sesión de entrevista, 27 de agosto de 2019)

En el contexto de las nuevas reformas de la Ley Universitaria, los estudiantes mostraron más interés en los acuerdos establecidos con Sunedu para mejorar la calidad de la educación, lo que involucra de más cerca al estudiante para participar en la gestión de la universidad. En este caso, observamos que la participación política se acompaña de intereses académicos con un gran impacto en los intereses de la comunidad universitaria, como es el caso de los comités de gestión:

La universidad se basa en ciertos principios, uno de ellos es el derecho de cogobierno, donde los estudiantes tenemos la opción y el derecho de poder participar en decisiones para la nuestra formación y, sin embargo, para la aplicación de esta ley se han venido vulnerando esos derechos, no tanto como, por ejemplo, como la elaboración de mallas académicas o ha afectado en que los estudiantes no tengan representación adecuada. (E5, sesión de entrevista, 27 de agosto de 2019)

\section{DISCUSIÓN}

El presente estudio tuvo como propósito describir la experiencia subjetiva de la participación política de un grupo de estudiantes de la UNMSM. Hemos explorado 
cómo se expresan sus vivencias alrededor de las temáticas de sentido de identidad comunitaria, empoderamiento y participación política.

Sobre la técnica de recolección elegida para este proceso, hemos corroborado en el trabajo de campo, y en la interacción con los escenarios sociales, qué vivieron los participantes (el conflicto del bypass de la avenida Venezuela) y cómo este contexto mantiene activos los comportamientos que subyacen a la participación política en la universidad, como una práctica cotidiana de la vida universitaria, la cual tiene diferentes expresiones. La interacción cara a cara con los estudiantes y la diversidad de reportes sobre su participación política fueron una oportunidad para revalorar la experiencia subjetiva desde la exploración individual psicológica.

De la revisión del marco teórico referencial sobre sentido de identidad comunitaria, y a la luz de los resultados obtenidos, coincidimos con Puddifoot, que esta orientación psicológica se hace manifiesta en un plano personal y en un plano de relación con los demás. Los testimonios nos han demostrado que, en la práctica de la participación política de estos estudiantes, los componentes del sentido de identidad se han reflejado en espacios de interacción académica, afectiva y social. El sentido de apoyo personal se ha manifestado en acciones de colaboración con sus pares que, mediante la participación política, evolucionan en actitudes de mayor compromiso (sentido activo de compromiso personal) cuando toman conciencia de que tan solo actuar habilita en ello competencias que antes no tenían:

Yo creo que recién me estoy dando cuenta qué puedo dar, quizás, una opinión más abierta porque creo que he sido pasiva en ese aspecto (...) Yo participando como centro, aparte ya una cosa más específica, yo creo que ha sido muy positiva, estoy muy feliz, por más que sea muy cansado, de haberme atrevido y de ayudar a muchos estudiantes y a muchas organizaciones también. (E3, sesión de entrevista, 16 de setiembre de 2019)

Lo deducido anteriormente, en diálogo con otro componente del sentido de identidad comunitaria, como es el sentido de contento personal, a nuestro entender, la piedra angular del sentido de comunidad general se convierte en un motivo para comprometerse cada vez más con su propia participación y la participación política de la comunidad que representa, a pesar de cómo se presenta la realidad inmediata en su organización:

Acá hay un Centro Federado en sociales pero que ha estado inactivo por dos años (...) La COGREE [Comisión de Gremios Estudiantiles] es la que está asumiendo, ha estado asumiendo por diversos problemas (...), problemas por votos por intromisión de compras de votos. Entonces, cada vez las votaciones se hacen inválidas. (E2, sesión de entrevista, 11 de setiembre de 2019) 
Este aspecto del sentido identidad que impulsa un mayor compromiso en la participación se conoce como el carácter motivador del compromiso, "lo que hace que una persona continúe un curso de acción cuando las alternativas negativas o positivas influyan a la persona para abandonar la acción", según Klinger (como se citó en Montero, 2011).

Del mismo modo que existe un contento por ser parte de la comunidad de estudiantes, observamos que la participación política que fortalece ese sentimiento también ha facilitado un empoderamiento, el mismo que se relata como aprendizajes o adquisiciones de nuevas habilidades para desenvolverse en la universidad, en esferas de lo académico y lo social. A su vez, se evidencia que este nivel de empoderamiento individual evoluciona a un empoderamiento organizacional, porque los estudiantes, como representantes estudiantiles, se posicionan en la esfera de la política interna de su facultad de estudios como participantes activos de un cogobierno que toma decisiones en el marco de sus necesidades como estudiantes:

Yo sé que, por ejemplo, en varias escuelas había representantes del Comité de Gestión (...) que ellos estaban, por ejemplo, en Lingüística, estaban apoyando a ver la malla curricular, la cual iba a cambiar, (...), o sea, qué cursos debe cambiar, qué curso no debe cambiar (...), en los Estudios Generales hay muchos electivos que no tienen nada que ver con las carreras que están llevando. (E3, sesión de entrevista, 16 de setiembre de 2019)

La participación activa en los comités de gestión valida la propia participación política en una esfera formal de decisiones, también denominada participación institucional, en términos de Delfino, Zubieta y Muratori (2013).

Sobre el empoderamiento a nivel comunitario, no podemos aseverar que la participación política de estos representantes estudiantiles tenga mayor influencia, ya que ellos mismos reconocen que su organización interna no es cohesionada. Algunos centros de estudiantes y centros federados, como primer nivel de organización estudiantil, no cuentan con representación estudiantil formal. Algunos cuentan con juntas directivas transitorias. Asimismo, existe una percepción generalizada de que a los demás estudiantes no les interesa participar de estos espacios. Es decir, el empoderamiento individual tiene un alcance en un empoderamiento organizacional, porque los representantes pueden participar en instancias de gobierno:

Me he dado cuenta la gran importancia, la gran necesidad que es estar ahí presentes como estudiantes en votos, porque ahí se, bueno, se abren comisiones. También participé en la comisión de nombramiento docente dentro de mi facultad, cuando era Centro Federado. (E7, sesión de entrevista, 12 de setiembre de 2019) 
Este actuar llega a impulsarse a un empoderamiento comunitario, un empoderamiento de la comunidad universitaria, donde diferentes actores, estudiantes representantes y no representantes, se vinculan de forma racional y afectiva en la acción de la participación política y de manera sostenible en el tiempo.

En esta reflexión también podemos afirmar que, en la actualidad, identificar en la comunidad universitaria en San Marcos una relación estrecha entre participación política y empoderamiento comunitario, como fenómenos, es bastante lejano, sobre todo porque existen elementos internos y externos que afectan directamente la experiencia subjetiva de sus estudiantes en general. Estos elementos internos tienen que ver con lo que han afirmado los entrevistados, como son las respuestas represivas por parte de sus autoridades a su participación política. "Pero las autoridades a veces hacen leyes, lo único que han hecho, que nos enteramos hace un mes, es que pretendían criminalizar la dirigencia estudiantil. Está sancionado con la expulsión de la universidad" (E1, sesión de entrevista, 26 de agosto de 2019).

Sabucedo (como se citó en Sandoval et al., 2018) señala que los elementos externos están asociados a la estigmatización social sobre su participación política no convencional, que es expuesta por los medios de comunicación y con la que los mismos estudiantes se han sentido impactados desde su primer año de estudios y durante el transcurso de su formación como estudiantes de una universidad pública:

En la UPC, cuando comenté por primera vez si soy dirigente a mis compañeros, mis amigos, "qué es eso, por qué te has metido ahí, vas a perder clases o eres roja, eres de izquierda", (...) "eres una roja o no sé, terrorista", (...) esta estigmatización de que un representante estudiantil es alguien que lucha por sus derechos, (...), que tiene que ser de provincia y que es este pobre, no, esos son la principales estigmatizaciones que lo tienen. (E9, sesión de entrevista, 7 de enero de 2020)

El empoderamiento, que surge como una oportunidad de aprendizaje individual, asciende a una influencia de nivel organizacional interno de la comunidad universitaria (centros de estudiantes en las facultades), pero en el nivel comunitario se detiene. Una de sus principales formas de manifestación, como es la participación política, se convierte en una amenaza, donde el principal factor que influye en el logro de ese nivel es el estigma social. Por ello, la relación de los fenómenos de empoderamiento y participación política de los estudiantes, de acuerdo a los testimonios recogidos, es de influencia mutua y reciprocidad en el nivel individual y organizacional. En el nivel comunitario se requiere posibilitar mayores acciones en cantidad y calidad, para que este se pueda desarrollar. Según nuestro análisis, existe un respeto por los logros obtenidos en lo concerniente a la participación política en la UNMSM, como ser reconocidos como representantes estudiantiles según el estatuto de la universidad, o mediante el ejercicio del derecho al cogobierno en 
sus facultades. Acciones que incrementen el empoderamiento comunitario deberían surgir de establecer puentes de comunicación con estudiantes de diferentes carreras, de tal modo que la comunidad de estudiantes se autorreconozca mediante esa red de contactos y pueda compartir sus necesidades en común, así como reflexionar sobre los recursos con los que se cuenta, para utilizarlos de acuerdo con el contexto que nos toque vivir.

Hablar de participación política en el contexto de la universidad es prestar atención a sus diversas expresiones, o clasificar de otra forma más específica cómo se han manifestado en diferentes espacios. Creemos que la acción de involucrarse con una representación estudiantil, como el centro de estudiantes, conlleva a pensar que los estudiantes participan políticamente al mismo tiempo en espacios convencionales y no convencionales. El solo acto de decidir asumir un cargo de compromiso frente a una comunidad ya significa una expresión de participación política. Así también, tenemos las acciones que conllevan a compartir su propia experiencia, como en esta investigación. Esto significa dar a conocer sus vivencias, es decir, lo que significa para ellos la participación política.

En alusión a los antecedentes citados, podemos coincidir en que la participación política puede ser tipificada en relación con un enfoque legal, porque en los testimonios se corrobora que, en el presente, la participación política convencional de los estudiantes es la más reconocida y apremiante para su empoderamiento individual y organizacional. La acción política no convencional aún se percibe como negativa, al menos en el ámbito físico.

A modo de aporte a la tipificación de la participación política, como hemos citado en las referencias consultadas, consideramos que, desde las vivencias autorreportadas por los estudiantes representantes estudiantiles, la participación política que llevan a cabo es una sola, y esta se expresa de diferentes maneras. En otros términos, en la participación política que ejercen en la universidad, no diferenciada en su experiencia, no se identifica una intencionalidad de ser convencional o no convencional, según indica Sabucedo (como se citó en Sandoval et al., 2018). Desde sus propias experiencias, su accionar político se puede tipificar según el espacio de interacción en el que emerge (amigos, compañeros de facultad, docentes, autoridades) y según la expectativa o la influencia que esta pueda tener en lo individual, lo organizacional o toda la comunidad universitaria, que da como resultado un empoderamiento de la misma participación política del estudiante. Visto en un ciclo de relaciones: la participación política empodera a quienes la ejercen y, a su vez, motiva una mayor participación cuando el individuo toma conciencia de que sus acciones lo han empoderado en diferentes niveles.

Por último, si deseamos incluir, en un análisis integral, el papel que cumplen el sentido de identidad comunitaria, el empoderamiento y la participación política en la experiencia subjetiva de los estudiantes de la UNMSM, nos atrevemos 
a sugerir el siguiente esquema explicativo de la interdependencia de estas y el efecto individual que podría tener.

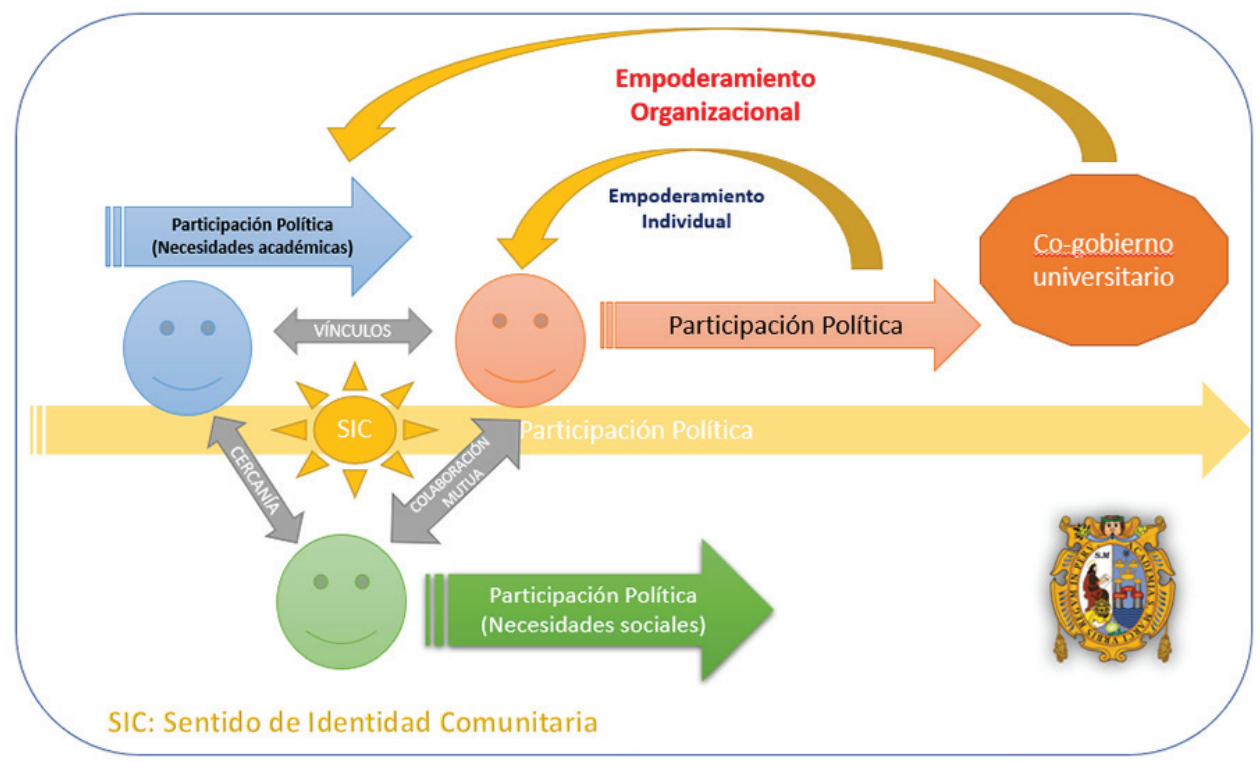

Figura 2. Esquema de relaciones entre sentido de identidad comunitaria, empoderamiento y participación política

\section{CONCLUSIONES}

Como parte de las conclusiones, vamos a valorar los aportes más relevantes de todo el proceso de esta fase de investigación, que a su vez forma parte de un estudio de mayor profundidad en el programa de doctorado de Psicología en la PUCP:

a. Las investigaciones acerca de la categoría participación política, en el campo de la psicología y desde una perspectiva cualitativa-fenomenológica, aún se encuentra en una fase incipiente en el contexto peruano. Se considera una tarea pendiente de exploración y comprensión profunda, ya que en la población de estudiantes de universidades públicas se ha indagado sobre la política y su ejercicio, sin prestar atención a los efectos psicosociales de esta acción en los individuos y las comunidades que representan en la universidad.

b. El sentido de identidad comunitaria motiva a los estudiantes a involucrarse en una participación política más activa, y se percibe como una necesidad compartida con su comunidad de estudiantes, que genera vínculos y, además, produce aprendizajes y satisfacciones tanto para el individuo como para el grupo que representa. 
c. Se observa que la mayor diversidad de experiencias de participación política refuerza el vínculo de cercanía y colaboración de los representantes estudiantiles con sus pares en la universidad.

d. El empoderamiento de los estudiantes entrevistados se refleja en los niveles individual y organizacional, de acuerdo con lo propuesto por la teoría multinivel de Zimmerman. De las vivencias escuchadas y el contexto en el que se realizó el estudio, se concluye que el nivel de empoderamiento comunitario se expresa en algunas situaciones circunstanciales, cuando surgen prácticas no convencionales de ejercer participación, como la toma de la universidad (8 de enero de 2020). En esta situación, se pudo atestiguar una expresión del sentido de unión de la comunidad universitaria, en la que se incluyen otros actores, como docentes y trabajadores, para permitir una única acción que involucre a la comunidad de estudiantes.

e. La participación política de los representantes de los Centros de Estudiantes de la UNMSM se expresa de diferentes maneras, de acuerdo con los espacios de interacción con otros actores de la comunidad universitaria, y con las expectativas de su propia experiencia de participación política en relación con el empoderamiento individual y organizacional, logrado en un espacio y tiempo definido.

\section{Agradecimientos / Acknowledgments:}

A los estudiantes y autoridades de la UNMSM.

\section{Fuentes de financiamiento / Funding:}

Recursos propios.

\section{Rol de los autores / Authors Roles:}

JEQ: investigador y autor principal del estudio.

MRZ: co-autora del artículo.

\section{Aspectos éticos / legales; Ethics / legals:}

Hemos cumplido las normas éticas de investigación contando con las autorizaciones y consentimientos informados los cuales tenemos bajo resguardo.

\section{Conflicto de intereses / Competing interests:}

Declaramos que no hemos tenido conflicto de intereses. 


\section{REFERENCIAS}

Botero, P., y Torres, J. (2008). Perspectivas teóricas para comprender la categoría participación ciudadana-política juvenil en Colombia. Recuperado de http://www.scielo. org.co/pdf/rlcs/v6n2/v6n2a05.pdf

Braun, V., y Clarke, V. (2006). Using thematic analysis in psychology. Qualitative Research in Psychology, 3(2), 77-101. http://dx.doi.org/10.1191/1478088706qp063oa

Brussino, S., Rabbis, H., y Sorribas, P. (2008). Una propuesta de categorización de la participación politica de jóvenes cordobeses. Recuperado de https://www.researchgate.net/publication/228085301_Una_propuesta_de_categorizacion_de_la_participacion_politica_de_jovenes_cordobeses

Cano, A., Quiroz, M., y Nájar, R. (2017, 10 de enero). Jóvenes universitarios en Lima: política, medios y participación. Comunicar. Recuperado de http://repositorio.ulima. edu.pe/handle/ulima/4515

Córdova, C. (2013). Autoritarismo, actitudes y participación política no convencional (tesis de pregrado). Pontifica Universidad Católica del Perú, Lima, Perú.

Delfino, G., Zubieta, E., y Muratori, M. (2013). Tipos de participación política: análisis factorial confirmatorio con estudiantes universitarios de Buenos Aires, Argentina. Psicología Política, Associação Brasileira de Psicologia Política, 13, 301-318. Recuperado de http://pepsic.bvsalud.org/pdf/rpp/v13n27/v13n27a07.pdf

Fernández, I., Morales, J., y Molero, F. (Coords.) (2012). Psicología de la intervención comunitaria. Bilbao: Editorial Desclée de Brouwer.

Ferullo, A. (2006). El triángulo de las tres P. Psicología, participación y poder. Buenos Aires: Paidós.

Grimberg, M. (2001). Vih-Sida, vida cotidiana y experiencia subjetiva. Una revisión conceptual de las dimensiones de vivir con Vih. VI Congreso de Ciencias Sociales y Salud, Lima, Perú. Recuperado de http://amr.org.ar/amr/wp-content/uploads/2015/07/ n82a394.pdf

Hatibovic, F., Sandoval, J., y Cárdenas, M. (2012, diciembre). 'Posiciones de sujeto' y acción política universitaria: análisis de discurso de estudiantes de universidades de la región de Valparaíso. Última Década. Recuperado de https://dialnet.unirioja.es/ servlet/articulo?codigo $=4413279$

Hernández-Sampieri, R., y Mendoza, Ch. (2018). Metodología de la investigación. Las rutas cuantitativa, cualitativa y mixta. México D. F.: Mc Graw Hill Education.

Jave, I., Cépeda, M., y Uchuypoma, D. (2015). La acción política frente al estigma de la violencia entre los jóvenes universitarios posconflicto: los casos de la Universidad Nacional Mayor de San Marcos y la Universidad Nacional de San Cristóbal de Huamanga, Anthropologica. 33(34), 187-202.

Jorge, A. (2016). Relaciones entre ideología, participación y cinismo político en jóvenes de Lima (tesis de pregrado). Pontifica Universidad Católica del Perú, Lima, Perú. 
Kaliksztein, B. (2001). La experiencia subjetiva de la enfermedad en pacientes con esquizofrenia. Revista de Psicología de la PUCP. Recuperado de https://dialnet.unirioja.es/descarga/articulo/4531332.pdf

Katayama, R. (2014). Introducción a la investigación cualitativa. Fundamentos, métodos, estrategias y técnicas. Lima: Universidad Inca Garcilaso de la Vega.

Larkin, M., y Thompson, A. (2012). Interpretative phenomenological analysis. En A. Thompson, y D. Harper. (Eds.), Qualitative research methods in mental health and psychotherapy: a guide for students and practitioners (pp. 99-116). United Kingdom: John Wiley \& Sons. https://doi.org/10.1002/9781119973249

Ley n. ${ }^{\circ}$ 32220. Diario Oficial El Peruano, Lima, Perú, 9 de julio de 2014. Recuperado de http://www.sunedu.gob.pe/nueva-ley-universitaria-30220-2014/

Lozano, M. (2008). Los procesos de subjetividad y participación política de estudiantes de psicología de Bogotá. Diversitas: Perspectivas en Psicología, 4(2), 345-357. Recuperado de http://www.redalyc.org/articulo.oa?id=67940210

Martínez, M. (2004). Ciencia y arte de la metodología cualitativa. México D. F.: Trillas.

Ministerio de Educación. (2014). Jóvenes, universidad y política. Una aproximación a la cultura política juvenil desde las perspectivas de los estudiantes de la Universidad Nacional Mayor de San Marcos. SENAJU. Lima: MACOLE.

Montero, M. (2011). Introducción a la psicología comunitaria. Desarrollo, conceptos y procesos (4. ${ }^{\mathrm{a}}$ ed.). Buenos Aires: Paidós.

Montoya, L. (2016). Juventudes, políticas y poder en el Perú (tesis de maestría). Universidad Nacional Mayor de San Marcos, Lima, Perú.

Noreña, A., Alcaraz-Moreno, N., Rojas, J., y Rebolledo-Malpica, D. (2012). Aplicabilidad de los criterios de rigor y éticos en la investigación cualitativa. Aquichan, Vol. 12, No. 3, 2012, págs. 263-274. Recuperado de: https://dialnet.unirioja.es/servlet/ articulo?codigo $=4322420$

Nureña, C. (2016). ¿El estigma genera despolitización? Participación, estigmatización por la violencia política y rechazo a Sendero Luminoso en la UNMSM. Revista Andina de Estudios Politicos, 4(2), 117-133. Recuperado de https://www.researchgate.net/ publication/309763093_El_estigma_genera_despolitizacion_Participacion_estigmatizacion_por_la_violencia_politica_y_rechazo_a_Sendero_Luminoso_en_la_ Universidad_Nacional_Mayor_de_San_Marcos

Nureña, C. (2017). Juventud y cultura política en el Perú: El caso de los estudiantes de la Universidad Nacional Mayor de San Marcos de Lima. Revista Argumentos, 11(1), 60-64. Recuperado de https://revistaargumentos.iep.org.pe/wp-content/uploads/2017/06/NURENA_11_1_2017-1.pdf

Patton, M. (2015). Qualitative research and evaluation methods. 4a. Ed. Thousand Oaks: Sage Publications.

Penalva, C., Alaminos, A., Francés, F., y Santacreu, O. (2015). La investigación cualitativa: técnicas de investigación y análisis con Atlas.ti. Ecuador: PYDLOS Ediciones. 
Puddifoot, J. (2003). Exploring 'personal' and 'shared' sense of community identity in Durham City, England. Journal of Community Psychology, 31(1), 87-106. https://doi. org/10.1002/jcop.10039

Sánchez-Vidal, A. (2007). Manual de Psicología Comunitaria. Un enfoque integrado. Madrid: Pirámide.

Sandoval, J., Hatibovic, F., y Bobowik, M. (2018). Validación de la Escala de Tendencias de Acción Política en una muestra de estudiantes universitarios chilenos. Psykhe, 27(1), 1-17. http://dx.doi.org/10.7764/psykhe.27.1.1030

Silva, C., y Martínez, M. (2004). Empoderamiento: Proceso, Nivel y Contexto. Psykhe, 13(2), 29-39. https://dx.doi.org/10.4067/S0718-22282004000200003

Tarazona, D. (2004). Acercamiento a la experiencia subjetiva del voluntariado social en organizaciones juveniles. Dispersión, 1(3), 1-12. Recuperado de http://dds.cepal.org/ redesoc/archivos_recursos/231/3_tarazona.pdf

Vasilachis de Gialdino, I. (Coord.). (2014). Estrategias de investigación cualitativa. Barcelona: Gedisa.

Willig, C. (2013). Introducing qualitative research in psychology. London: McGraw-Hill Education.

Yarasca, R. (2019). Movimiento estudiantil universitario en el Perú de los 60. Lima: Editora Páginas. 
